\title{
Author-Driven Approaches to Computational Narrative Design for Games
}

\author{
David John Tree ${ }^{1}$, Alessio Malizia ${ }^{2}$ \\ ${ }^{1}$ Games and Visual Effects Research Lab, School of Creative Arts, University of \\ Hertfordshire, Hatfield, United Kingdom \\ ${ }^{2}$ School of Creative Arts, University of Hertfordshire, Hatfield, United Kingdom \\ d.tree@herts.ac.uk, a.malizia@herts.ac.uk
}

\begin{abstract}
Accessible Head Mounted Displays (HMD) have provided mass access to Extended Reality (XR) content as never before. One of the key complaints from HMD owners, however, is the lack of substantial high-quality content (Moore, 2017). Coupled with the domain-specific topic of presence, which describes a state beyond the concept of immersion instead with the user feeling part of the virtual world.

Utilizing traditional production techniques cost and the duration of the resultant product are inextricably linked, although some progress reducing manual hours has been made with the introduction of Procedural Content Generation (PCG); the focus of this shift has been on asset creation rather than narratives (Kapadia et al., 2017). While PCG can reduce the cost of production it does not however directly increase the duration of the game. The current solution from developers has been to implement game mechanics to increase the duration of the game, leading to a rise in arcade-style wave shooters (Anon., 2018), however, this solution is not applicable to long-form content such as narrative-based Role Playing Games (RPG), story-based first-person action games and interactive narratives.

The intended contribution of this paper is both to describe the challenges to the academic knowledge in procedurally generated content and computational narratives but is also about studying how those approaches can be democratized, enabling wider adoption within the content creation community, with a view to their eventual use within non-entertainment sectors.
\end{abstract}

Keywords: Interactive Narrative, Computational Narrative, Agency.

adfa, p. 1, 2011.

(C) Springer-Verlag Berlin Heidelberg 2011 


\section{Introduction}

While the introduction of fully automated and mixed-initiative design processes such as Procedural Content Generation (PCG) and Computational Narrative $(\mathrm{CN})$ can reduce the manual hours required to design and produce games, hitherto the impact of these techniques is predominated in the visual and special aspects of video games showing little impact in the players narrative experience. These techniques have demonstrated an ability to improve game duration and reduce overall production cost, however this has been to the detriment of narrative complexity leading to an increase in arcade and shoot-em-up style video games rather than an elaborated form of content such as Role-Playing Games (RPG), story-based first-person action games and interactive narratives which offer a better or more immersive experience.

Some approaches are available to improve the depth and breadth of games based on mixed-initiative interactive narratives but these have been seldom employed in game engines so far. Such approaches include combining Machine Learning techniques (supervised or unsupervised) with player analytics to model players' behaviour and adapt the storytelling accordingly. Furthermore, Visual Languages can be used to support authors/designers in modelling Non-Player Characters (NPCs) without requiring advanced programming skills.

We believe that by employing such techniques authors, players and producers can benefit in their respective activities, enabling a renewed focus on creativity rather than on technical aspects and requirements. With the end goal of increasing the complexity in the game narrative and therefore the sense of immersion in the game world along with increased productivity. Simultaneously using those approaches pose new research questions and challenges.

For instance, the multimodal possibilities offered by new technologies such as Augmented and Virtual Reality and Conversational Agents are limited if not integrated into the game narrative generating discontinuity in the sense of immersion and cognitive flow (Faiola et al., 2013). A crucial element of games is, for instance, the design of Non-Player Characters (NPCs), However, to improve NPCs behaviour full integration with the game narrative is needed, e.g. by allowing them to use elaborate strategies to achieve the game objectives in a sensible way.

In this paper, we will explore the field of automated and mixed-initiative design of game elements and interactive narratives, describing the state of the art and focusing on two very promising techniques: Procedural Content Generation and Computational Narrative with the aim of informing the community on the research questions and challenges posed by such approaches. We describe also the possibilities offered by such algorithmic approaches that can positively impact player experience, authors ability to focus on narratives and improve publishers/producers benefits in terms of production workflow, cost and duration. 


\section{Background}

\subsection{Interactive Narratives}

Although Non-linear storytelling is now synonymous with the interactive media, conceptually it has existed for as long as stories have been told. Where in person we might pass some information on to someone else by way of a story, we the storyteller will judge our audience and adapt the story accordingly rather than providing a verbatim account of events.

The introduction of the print media changed this aural tradition to one of accurate, complete reproduction thus changing the passage of information forever. However the yearning for adaptive narratives made its way into printed media, early examples of this non-linear storytelling can be found in the Choose Your Own Adventure (Montgomery, Peguy and Cannella, 1982) series of books part of a genre of fictional books which enable the reader to select the path and therefore the outcome of the story. This form of interactive fiction is defined as a branching narrative as when plotted the narrative would show a tree-like structure. Fighting fantasy (Jackson and Livingstone, 1982) later combined the mechanics of Tabletop Role Playing Games (TRPG) such as the popular dungeons and dragons (Gygax and Arneson, 1974) and through this combination, both a feeling of player presence and reader agency was achieved with the added dimension of alea (Caillois and Barash, 1961) based gameplay. To achieve this the books included integral character sheets which stored information of previous decisions and the current character status.

Interactive narratives enable the reader limited agency over the narrative by offering the reader a choice at key points throughout the story, thus allowing the reader to select the outcome of the story. Although this agency was initially limited through the printed form it was later explored through hypertexts to enable a more fluid experience as explored by Aarseth (1997).

Due to their printed nature existing artefacts in this field require an entirely manual approach to authoring thus the authoring workload increases exponentially with the number of choices available to the player. Ryan (Marie-Laure Ryan, 2009) suggests that the holy grail of interactive narratives is the freeform provided by dynamic creation of a narrative within the defined story world. Ryan has long been an investigator within the field of interactive narratives since her acclaimed work "Hamlet on the holodeck" (Murray, 1998).

Literature Role Playing Games (LitRPG) is a sub-genre of fiction whereby the protagonist takes part in a Role-Playing Game either digital or tabletop, exploring the character's exploits, with a common trope of highlighting the game mechanics of the world indicating gained experience or achievements mirroring that found within real games. Although not interactive themselves this literary form provides a space for the examination of ethics, methods of interaction and more recently the interaction of human characters with the system. The advantage of this hypothetical exploration is the lack of the implementation stage needed to explore these scenarios in a real game, permitting thinking beyond current systems available and inspiring real-world development. 
In the Awaken Online LitRPG series (Bagwell, 2016) players partake in a new Virtual Reality Massively Multiplayer Online Role-Playing Game (MMORPG) entitled "Awaken Online" where the Game Manager named Alfred controls not only the ongoing story but the in-game gods and NPC behaviours. The books raise several questions within the VR arena including the ethics of high gore games and the possibility of simulation induced Post Traumatic Stress Disorder as well as the possible therapeutic effects of the simulation environment. More relevant to this paper Bagwell explores the role of an A.I. with unclear directives experimenting to encourage players to engage for longer. Although the anthropomorphized Alfred is portrayed as a sentient intelligence with the Bagwell referencing that the in-game AI had passed the Turing test (Turing, 1950) the question of how the success of gameplay and interactive narratives is very relevant and we explore this question in the computational narrative section of this paper.

Continued development of interactive technologies and distribution mediums have led to the inception of Interactive Cinema and TV such as the recent Bandersnatch, a psychological thriller interactive film in the science fiction anthology series Black Mirror made available on streaming by Netflix (Slade, 2018). In this interactive film, the audience is forced to make decisions for the protagonist, the designer has encouraged impulse driven response through the application of a time window to provide a choice (ten seconds). The designers provide the viewers with five main endings with slightly different variants within each ending while making use of loops to cull extraneous story elements.

However, the interactive video technologies are still hampered by the pregenerated necessity of the delivery platform, and while some efforts to enable true agency have been made through simultaneous production and viewing (Weber, 2016; Montazemi, 2017) these only apply to a niche audience and are inaccessible to the general public.

Video games offer a unique environment for the exploration of Artificial Intelligence and more specifically Interactive Narratives as videogame environments are data rich and optimised for the exploitation of interaction (Yannakakis and Togelius, 2018). Video games developers can be broadly categorised as either high budget mainstream (AAA) and independently funded studios (Indie) AAA studios have implemented interactive narratives within some titles such as Shadows of Mordor (Productions, 2014) which features an adaptive story world with its Nemesis game system, Allowed for the replacement of Non Playing Characters to be replaced through promotion of other NPCs. The developers combined this with the ability of NPC characters who have defeated the player in the past to remember the encounter and to taunt the player on the next instance.

However more ambitious solutions are currently being investigated by Indie studios driven by the need to create efficient methods for producing engaging longform content. Although in the past this been the downfall of titles which generated large followings within the gaming community such as the now infamous No Man's Sky (Murray et al., 2016) which was initially advertised to create a fully adaptive game space upon release the majority of the procedural content had been removed (Caldwell, 2016). This has not dissuaded other Independent studios from pursuing the 
objective of a fully procedural game space, more recently a crowd-funded title, Pine (Twirlbound, 2019), currently under development, proposes to implement a game world that adapts to the player, utilising a combination of generative algorithms.

\subsection{Computational Narrative}

The convergence of gestural, verbal and interactive media termed multi-modal interaction has become a possibility within recent years due to mass market access to VR motion controls for gestural input (Anon., 2012; Robertson, 2016a, 2016b) A.I. driven verbal interfaces (Solon, 2018) and high-powered home computing, enabling physically accurate rendering of a real-time worlds. With the platforms becoming increasingly stable and defined the prime opportunity for content creation has arrived. As this multi-modal platform matures, we will be investigating combining these systems with computational narratives to enable for the creation of adaptive narrative systems. The following section focuses on current developments in Computational Narratives and later the more specifically on the application of $\mathrm{CN}$ within the context of video games.

In their survey, Yannakakis and Togelius (2015) provide a structured approach to the alignment of Artificial Intelligence and Machine Learning Techniques to the applications of these techniques within the video-game domain, demonstrating the reach of the A.I. beyond controlling Non-Playing Characters (NPCs) and identifying gaps in the convergence of these research areas. Although the lack of convergence could be down to inherent incompatibilities of the techniques with the target area, the overall conclusions presented reinforce the need to further investigate linkages between the different areas of $\mathrm{AI}$ and ML within the domain of games.

As a subfield of Artificial intelligence, Computational Narrative aims to imbue computational systems with story intelligence, providing those systems with the ability to understand and synthesise information expressed in a narrative form. For the purposes of exploration, this field is often divided into two distinct areas, story analysis and story generation.

Story analysis combines techniques in Natural Language Translation, Sentiment analysis and applies them to a corpus of stories to gain insights into the structures, meaning and emotions expressed with a view to distilling the key structures of stories into an interpretable form.

Vonnegut, K (2004) posited that all stories have shapes, by plotting the happiness of the protagonist along the $\mathrm{Y}$-axis of Good-fortune and Ill-fortune against an $\mathrm{X}$-axis of beginning to end. This hypothesis has been later ratified by the work of Reagan et al. (2016) developers of the Hedonometer which utilises a big data approach to story analysis applying sentiment analysis to the Project Gutenberg Corpus. Novel to these approaches is the focus on the emotions of the characters rather than to the plot itself, as our focus is on the prescience of the player within the game world and the purpose of the story is to impress emotions, therefore it is vital that we understand the emotional states of both NPCs and possessed characters alike.

In their survey of story generation techniques for authoring computational narratives Kybartas and Bidarra (2017) outline the field of story generation placing 
papers on a scale comparing input from space automation and plot automation to the generated narrative. They identify that further investigation is warranted into the field of automated space generation which would contravene traditional practice of narrative design where the author designs the world/space before the creation of the story within the world. The authors of this paper also identify that the field of computational narrative needs a standard model of computational narrative that would allow the myriad of bespoke systems to interact to form a succinct narrative, these conclusions are supported by a significant quantity of background research the majority of which is published in subject-specific journals.

Li et al.(2013) pose to address the challenge of story generation within an unknown domain, the paper concludes that, by crowdsourcing the source corpus, their system can generate stories of equal quality to that of untrained humans, this is supported by testing that was run on a sample of 60 participants who were paid to provide feedback on the story generated by the plot graph; the data supports the conclusion that the system has successfully generated a storyline which required a similar level of correction to the human counterpart. It should be noted that the testers were paid, and the experiment was undertaken using Amazon Mechanical Turk which in its nature subsets the population used and may not be representative of culture as a whole. The conclusions drawn are influential in that they present a working model for resolving the knowledge-intensive training phase of story generation in an affordable and innovative way.

As alluded to earlier, the field of computational narratives is been divided leading to the separate representation of story elements. Valls-Vargas, Zhu and Ontañón, (2017) illuminate this dysfunction between generative and analytical models of computational narrative. Later highlighting the lack of an end to end solution, that would enable the introduction of a partially completed text with the output of a completed narrative with the system having prior knowledge of domain-specific areas. The paper concludes with the presentation of a prototype combined system; combined systems such as this will be vital to the enabling of unsupervised machine learning approaches allowing the system to both generate and evaluate the efficacy of the narratives.

Horswill, Montfort and Young (2014) provide an overview of papers covered in the 2015 Transactions on Computational Intelligence and AI in games. Concluding that some progress has been made in the field of AI in games and some commercial examples have started to appear. A key challenge in the field is disjoint between the research community and content creators. Highlighting the fear that if the field continues to progress along its current trajectory the AI techniques will continue to be aesthetically unproductive. This argument is supported through the representation methodologies used to analyse the success or failure of the AI systems which are currently predominated by HCI (Human-Computer Interaction) methodologies rather than methodologies utilised by the arts. The conclusions drawn in this paper support the need for a change in the methodology for the analysis and scoring of narrative generation systems to better accommodate aesthetic values.

Riedl, M (2012) outlines the field of AI in Video Games, specifying four fields of research: Human Level AI, Better Games, Supporting Game Development Practices 
and New Experiences before identifying the open problems in interactive narrative. A common theme throughout the challenges is the need for further research into the methods and techniques of autonomous drama manager's which take the traditional tabletop games role of dungeon master. Outside the field of AI Riedl highlights the success of applying psychology aware algorithms to guide players through the game world, mitigating players awareness of the game master.

Inspired by Table-top Roleplaying Games (TRPG) Ono and Ogata present a combined system approach to Narrative generation within a game environment through the combination of an Integrated Narrative Generation System (INGS) into the Automated Narrative Generation Game (ANGG). Novelty is found in the use of computer-controlled Game Manager (GM) and Players (PL) in automating both actors within the game, world collaboration is formed where the Game Manager generates the high-level story world and the Players propose enhancements on the low level. In addition to this, the techniques used for the low-level micro level control of the representation are founded on the techniques used in the marketing and film industries making it relevant to this work.

\subsection{Agency.}

Agency is the ability of a player to have an impact on the game world, as highlighted by Harrell, D and Zhu, J (2009) often this is misconceived to be the ability of the player to have sole and unencumbered free will. However, Harrell et.al. propose that instead agency should be considered in three forms: player agency, system agency, and authorial agency. This theory is supported through the analysis of contemporary media artefact's which align to these concepts and with reference to the mechanics of the real world. While the objective in creating the game world, is not to wholly replicate real-world rules, for the maintenance of the suspension of disbelief a plausible reality is required. Building on this theory of split agency games the memory, reverie machine (Zhu and Harrell, 2009), explores this hypothesis through an experimental AI-driven game of agency where the balance of agency is dynamically adapted during gameplay.

Within the context of the game world, system agency can be considered the application of the rules and mechanics of the game, an integral part of the play. The interesting element here is that the application of agency cannot simply be evaluated as a temporal control of an event where a single actor (player, system or author) has control over the current state of the game world. Instead agency should be considered for each element that is controllable, for example in the case of the game cinematic the author has control over the location of a player and the story unfolding around them, however most games will allow the player agency over the player viewport especially in the case of XR content where the restriction of head movement can increase the chances of simulation sickness. (Hettinger and Riccio, 1992)

Authorial agency is limited by two factors, that of how the CN system encodes the authors narrative template and how the $\mathrm{CN}$ system interprets the author's template to generate the game narrative. However, before these can be investigated, a representational model for computational narratives needs to be agreed. Initial 
research into this field has revealed that some attempts have been made to move the representational model of computational narratives beyond that of Hierarchical node trees and Directional Acyclic Graph. Such as the model presented by Verbrugge (2003) which combines Petri Nets with bi-directional edge flow to present a hypergraph representation of narrative.

Alternative models for representing these narratives can be seen in the system developed by Poulakos et al. (2016) who have begun to address the democratisation of computational narrative intelligence through the development of a graphical tool integrated into Unity3D games engine. The evidence presented is a small-scale test of computer science students who are inherently and more technically minded, somewhat limiting the validity of the results drawn; nevertheless, the objective of the study was an initial indication of the system and to receive feedback to support additional work. While the conclusions drawn are not in themselves of surprise, indeed implementing a graphical user interface improves accessibility to complex computational systems, the solution itself and the objectives of the paper are aligned with our objectives and one of the possible areas identified for development of a 3D interface for the story world generator is of interest in respect of our identified research question.

\section{Research Questions}

Through the exploration of existing approaches reported in the literature and adopted by the games industry, we have identified the following open questions relevant to addressing. Furthermore, two methodologies appear to be more adequate to tackle the following research questions to improve the end-users experience: Procedural Content Generation and Computational Narratives. Such approaches present a promising solution but also some quite relevant challenges for the field.

We will focus on three categories of potential end users: authors (e.g. game and narrative designers), players and producers/publishers. Our research questions therefore will be:

1. How can Computational Narratives and Procedural Content Generation assist authors in the design of interactive narratives so that players will have a better experience and producers could benefit by such approaches?

2. How can Computational Narrative and Procedural Content Generation techniques be integrated into existing middle-ware / game engines to enable the democratization of such technology, supporting authors in their narrative design approach and providing a better player experience?

3. How is the feeling of presence impacted by the integration of a computationally generated interactive narrative to improve the quality of player experience, stimulate authors creations and enable game publishers to streamline their production? 


\section{Challenges}

Procedural Content Generation (PCG) is employed in the automatic or semiautomatic design and generation of game elements from level maps to missions and textures. More recently, several approaches and solutions emerged on the market that show promising contributions to the fields of PGC and $\mathrm{CN}$, such as computational creativity, end-user development and interaction design.

Computational Narrative $(\mathrm{CN})$ takes a different but equally promising approach: focusing on story creation through the modelling of existing stories before applying generative algorithms to synthesise new narratives within the same domain. Stories are crucial to provide player's immersion and feeling of suspension of disbelief contributing to the effective and cognitive dimensions of the player's experience.

A combination of these two approaches can be effective in tackling the research question (1): How can $C N$ and PGC assist authors in the design of interactive narratives so that players will have a better experience and producers could benefit by such approaches?

PCG can be implemented in form of AI-assisted design tools which assist authors in generating game content: for instance, tools can support the creation of level maps by employing constraints inspired by the quality of player experience. Through the application of supervised machine learning, players can be involved in the co-design of level maps that can be used to train a machine learning algorithm to model constraints based on player's categories as in Bartle's taxonomy of player types (Bartle, 1996) and therefore providing an AI-assisted PCG tool. Assuming the authors are non-programmers a visual language can be used to enable an AI-based PCG approach to the design and generation of game levels (Shapiro and Ahrens, 2016) Furthermore, producers can benefit by integrating PCG into their production pipeline optimising cost and time to market.

Computational Narrative can provide support to authors in form of tools for generating narratives, such as drama management tools (Mateas and Stern, 2003). CN tools need further exploration since few are available and are mainly language-based as in textual adventures where those tools can generate interactive fiction. With the advent of multi-modal interactions more advanced computational narrative tools are needed to take advantage of such technologies and provide more advanced player experiences. Hypermedia visualisation tools in utilising a visual platform will provide great insight in the design of advanced multidimensional narratives (Charles et al., 2011). For instance, first-person narratives might benefit from the so-called believable agent's where NPC (Non-playing Characters) can render more realistic behaviour following the story pattern perhaps modelled on human player data collected while in game. Producers can benefit from $\mathrm{CN}$ by integrating a more immersive narrative in their games taking advantage of multi-modal interaction from the very beginning of story authoring in addition to game replay value with new avenues for Downloadable Content (DLC). 
While PGC and CN provide a promising approach to the adoption of interactive narratives in games some challenges are still to be addressed to fully employ such approaches:

- How to design Visual Languages or Visual Tools that can help authors design and generate complex and multi-modal interactive narratives.

- How to co-design game elements with players to train AI-assisted tools to support PCG and CN for non-programmers (e.g., authors or designers)

- How to employ Hypermedia visualizations to integrate complex narratives in gameplay.

For such approaches to have an impact in the interactive narrative and game areas PCG and CN should not be restricted to academic research or early adopters, leading to our next research questions (2): How can Computational Narrative and Procedural Content Generation techniques be integrated into existing middle-ware / game engines to enable the democratization of such technology, supporting authors in their narrative design approach and providing a better player experience?

While PCG has been explored in research, as per evidence from our literature review it has been seldom employed in the mainstream design of games. Content creation software and middleware utilise scripting to automate content generation: e.g. MEL or Python scripting used in Maya to generate content or C\# as a scripting language for Unity. Efforts are required from authors to learn to programme, with such environments normally offering APIs (Application Programming Interfaces) but rarely user-friendly visual editors or visual tools. PCG can be used to generate different game elements such as level maps or even quests but such high-level activities will require programming. To allow authors to use PCG in existing game engines means to improve the player experience by allowing authors to focus on the narrative and not on other skills that they might lack. Furthermore, PCG could offer a way of modelling the quality of experience allowing simulation-based testing integrated into game engines, e.g. testing author-driven automatically-generated content by playing the game through a simulated player (an agent). At the same time, such an approach could open game design to authors focused on the narrative more than on the technology to implement it.

Computational Narrative can provide integrated storytelling workflows and patterns for existing game engines allowing the authors to take full advantage of the technical features offered by such middleware. By integrating Computational Narrative in existing game engines NPCs could become more proficient when developed as characters in the narrative using existing storytelling elements, e.g. elements of the Propp's functions (Imabuchi and Ogata, 2012) can be modelled in the Computational Narrative aspects of an existing game engine. Taking advantage of the multi-modal interaction features offered by modern game engines agents integrated into a story generated by Computational Narrative could be more realistic and proficient being able to integrate well in the narrative and become more believable improving the player experience. 
How to integrate PGC and $\mathrm{CN}$ approaches in mainstream game engines to improve the democratization of advanced narratives present some interesting challenges:

- How can game engines provide support to PGC and CN providing authors with user-friendly tools to generate content and design narratives?

- Can we identify open standards to generate game elements and narratives that can be supported by existing game engines?

- Can common narrative elements providing a better player experience be modelled in current game engines in a user-friendly way freeing the authors from technicalities and allowing them to focus on the narrative and player experience?

PGC elements and Computational Narrative must generate believable and immersive content to improve the player's experience and provide game producer with the ability of exploit multi-modal technologies (3): How is the feeling of presence impacted by the integration of a computationally generated interactive narrative to improve the quality of player experience, stimulate authors creations and enable game publishers in streamlining their production?

PGC can generate NPCs behaviour and interactions with game levels and actual player. Computational player modelling could learn NPCs behaviour to react consistently to human players. Machine learning algorithms for modelling NPCs could provide a better sense of presence, immersion and multi-modal experience for the players, e.g. the well-known limitation of NPCs in VR environments incapable of looking at player's eyes during dialogues.

Computational Narrative techniques could assist in generating more believable NPCs behaviour when interacting with a player following storytelling patterns. Agents (NPCs) able to plan actions according to a strategy and express behavioural and emotional attitude can offer a better player experience with respect to believability. Looking into integrating results from interactive virtual and conversational agents as well as humans might benefit the feeling of presence and realism of games highly improving the quality of player experience.

How to generate more believable and immersive experiences through PGC and CN approaches provide some interesting challenges:

- Can we employ machine-learning algorithms to model NPCs reactions to actual players' behaviour?

- Can we model players by learning their behaviour from data and patterns accumulated during game plays and inform interactive storytelling and automatically-generated agents to be more believable and thus offer a better player's experience?

- Could we take advantage of existing studies on conversational agents and chatbots to generate more realistic NPCs and improve the player feeling of presence and immersion in games and interactive narratives? 
We believe that the challenges identified in this section can be a source of inspiration for future research on author-driven approaches to computational narrative design for games with the aim of improving player experience, authors ability to focus on creativity and publisher's workflow and production management and optimisation.

\section{Conclusion}

As Alister E. McGrath (2014) stated referring to C.S. Lewis (Author of the Chronicles of Narnia): "Lewis wanted us to understand that the inner world is shaped by stories." The most relevant gaming and interactive stories experiences are based on complex narratives requiring a huge investment from game producers and publishers. Lately, with the rise of AI-oriented applications, Procedural-Generated Content (PGC) and Computational Narrative $(\mathrm{CN})$ aided stakeholders involved in the creation of interactive experiences. By allowing the author/designer to focus on the narrative and creativity while at the same time increasing productivity, such tools are becoming more and more crucial for interactive, multimodal experiences and game production. Nevertheless, such approaches require integration into existing production pipelines ensuring they improve the player/user experience. Without the utilisation of such techniques, fiscal pressures on game development have led to the dwindling of narrative lead immersive content leading to domination of shoot-em-ups and wavebased arcade shooters.

In this paper, we described some of the existing approaches and the research questions to inform the community of the possibilities and challenges posed by such approaches. Clearly, a multidisciplinary approach is needed to design tools to adequately support both designers and authors in the production of complex narratives within a mixed-initiative approach, disciplines such as artificial intelligence, end-user programming, information visualisation and user experience are just a few examples of the areas that might be involved in tackling such questions and challenges. We believe that PCG and CN can positively impact, not only the production costs and quality of future games and interactive experiences but also the author's abilities to focus more on creativity and less on technical skills while at the same time providing a better player experience.

\section{Future Work}

We plan to investigate towards a unified dynamic representational system for interactive narratives within games, using a measure of narratological and ludic success combined with a user interface design measure of success for the author's interaction with this new model. The aim will consists of improving the efficiency of generating immersive narratives that are functionally interactive with a focus on player agency. 
Acknowledgements. The Games and Visual Effects Research Lab (G+VERL) is funded by the European Regional Development Fund: Interreg: North Sea Region, Create Converge project

\section{References}

1. Aarseth, E. J. (1997) Cybertext: perspectives on ergodic literature. Johns Hopkins University Press.

2. Anon. (2012) Leap Motion Unveils World's Most Accurate 3-D Motion Control Technology for Computing - Leap Motion. Available at: https://www.leapmotion.com/news/leap-motion-unveils-worlds-most-accurate-3-d-motioncontrol-technology-for-computing (Accessed: 29 May 2018).

3. Anon. (2018) Time Carnage - More than just another wave shooter |VR Game Rankings: Reviews - Previews - Lists, $V r$ Game Rankings. Available at: https://www.vrgamerankings.com/single-post/2018/04/18/Time-Carnage---More-than-justanother-wave-shooter (Accessed: 31 May 2018).

4. Bagwell, T. (2016) Awaken online. Book 1 : catharsis.

5. Bartle, R. (1996) 'Hearts, clubs, diamonds, spades: Players who suit MUDs', Journal of MUD research, 1(1), p. 19.

6. Caillois, R. and Barash, M. (1961) Man, play, and games. Paris: Librairie Gallimard. Available https://books.google.co.uk/books/about/Man_Play_and_Games.html?id=bDjOPsjzfC4C\& printsec $=$ frontcover\&source $=\mathrm{kp} \_$read_button\&redir_esc $=\mathrm{y} \# \mathrm{v}=$ onepage \&q $\& \mathrm{f}=$ false.

7. Caldwell, B. (2016) The Broken Promise Of No Man's Sky And Why It Matters, Rock paper shotgun. Available at: https://www.rockpapershotgun.com/2016/08/17/brokenpromises-of-no-mans-sky/.

8. Charles, F. et al. (2011) 'Timeline-based navigation for interactive narratives. In Proceedings of the 8th International Conference on Advances in Computer Entertainment Technology', in 8th International Conference on Advances in Computer Entertainment Technology. ACM, p. 37.

9. Faiola, A. et al. (2013) 'Correlating the effects of flow and telepresence in virtual worlds : Enhancing our understanding of user behavior in game-based learning', Computers in Human Behavior, 29, pp. 1113-1121. doi: 10.1016/j.chb.2012.10.003.

10. Gygax, G. and Arneson, D. (1974) Dungeons and Dragons. TSR.

11. Harrell, D. F. F. and Zhu, J. (2009) 'Agency play: Dimensions of agency for interactive narrative design', Proceedings of the AAAI 2009 Spring Symposium on Narrative Intelligence II. Menlo Park: AAAI Press. aaai.org, pp. 44-52. Available at: http://www.aaai.org/Papers/Symposia/Spring/2009/SS-09-06/SS09-06-008.pdf.

12. Harrell, D. F. and Zhu, J. (2009) 'Agency Play: Dimensions of Agency for Interactive Narrative Design', Intelligent Narrative Technologies II - Papers from the AAAI Spring Symposium, SS-09-06, pp. 44-52. Available at: http://www.aaai.org/Papers/Symposia/Spring/2009/SS-09-06/SS09-06-008.pdf.

13. Hettinger, L. J. and Riccio, G. E. (1992) 'Visually Induced Motion Sickness in Virtual Environments', Presence: Teleoperators and Virtual Environments, 1(3), pp. 306-310. doi: 10.1162/pres.1992.1.3.306. 
14. Horswill, I. D., Montfort, N. and Young, R. M. (2014) 'Guest editorial: Computational narrative and games', IEEE Transactions on Computational Intelligence and AI in Games, 6(2), pp. 93-96. doi: 10.1109/TCIAIG.2014.2325879.

15. Imabuchi, S. and Ogata, T. (2012) 'A Story Generation System Based on Propp Theory: As a Mechanism in an Integrated Narrative Generation System', in. Springer, Berlin, Heidelberg, pp. 312-321. doi: 10.1007/978-3-642-33983-7_31.

16. Jackson, S. and Livingstone, I. (1982) The Warlock of Firetop Mountain. Available at: https://www.amazon.co.uk/Warlock-Firetop-Mountain-Fighting-

Gamebook/dp/1840463872/ref=oosr (Accessed: 25 May 2018).

17. Kapadia, M. et al. (2017) 'Computational narrative', in ACM SIGGRAPH 2017 Courses on - SIGGRAPH '17, pp. 1-118. doi: 10.1145/3084873.3084931.

18. Kybartas, B. and Bidarra, R. (2017) 'A Survey on Story Generation Techniques for Authoring Computational Narratives', IEEE Transactions on Computational Intelligence and AI in Games. ieeexplore.ieee.org, 9(3), pp. 239-253. doi: 10.1109/TCIAIG.2016.2546063.

19. Li, B. et al. (2013) 'Story Generation with Crowdsourced Plot Graphs', Proceedings of the Twenty-Seventh AAAI Conference on Artificial Intelligence. aaai.org, pp. 598-604.

20. Marie-Laure Ryan (2009) 'From Narrative Games to Playable Stories: Toward a Poetics of Interactive Narrative', StoryWorlds: A Journal of Narrative Studies, 1(1), pp. 43-59. doi: 10.1353/stw.0.0003.

21. Mateas, M. and Stern, A. (2003) 'Façade: An experiment in building a fully-realized interactive drama', in Game Developers Conference, pp. 4-8.

22. McGrath, A. (2014) If I Had Lunch with CS Lewis: Exploring the Ideas of CS Lewis on the Meaning of Life. Tyndale House Pub.

23. Montazemi, P. T. (2017) 'Device and method for playing an interactive audiovisual movie'. Available at: https://patents.google.com/patent/US20180090177A1/en (Accessed: 29 January 2019).

24. Montgomery, R. A., Peguy, L. and Cannella, M. (1982) The abominable snowman. Chooseco.

25. Moore, T. P. (2017) What Steam's Data Reveals About The Health of VR's Ecosystem VRFocus, vrfocus. Available at: https://www.vrfocus.com/2016/07/what-steams-datareveals-about-the-health-of-vrs-ecosystem/ (Accessed: 31 May 2018).

26. Murray, J. H. (1998) Hamlet on the holodeck: the future of narrative in cyberspace. MIT Press. Available at: https://mitpress.mit.edu/books/hamlet-holodeck (Accessed: 25 May 2018).

27. Murray, S. et al. (2016) 'No Man's Sky'. Hello Games. Available at: https://www.nomanssky.com/.

28. Poulakos, S. et al. (2016) 'Evaluating accessible graphical interfaces for building story worlds', Lecture Notes in Computer Science (including subseries Lecture Notes in Artificial Intelligence and Lecture Notes in Bioinformatics). Springer, 10045 LNCS, pp. 184-196. doi: 10.1007/978-3-319-48279-8 17.

29. Productions, M. (2014) 'Shadows of Mordor'. Warner Brothers.

30. Reagan, A. J. et al. (2016) 'The emotional arcs of stories are dominated by six basic shapes', EPJ Data Science, 5(1). doi: 10.1140/epjds/s13688-016-0093-1.

31. Riedl, M. O. (Georgia I. of T. (2012) 'Interactive Narrative: A Novel Application of Artificial Intelligence for Computer Games.', Proceedings of the 26th AAAI Conference on Artificial Intelligence, pp. 2160-2165. Available at: http://www.aaai.org/ocs/index.php/AAAI/AAAI12/paper/download/5153/5388. 
32. Robertson, A. (2016a) HTC Vive review | The Verge, The Verge. Available at: https://www.theverge.com/2016/4/5/11358618/htc-vive-vr-review (Accessed: 29 May 2018).

33. Robertson, A. (2016b) Oculus Touch review: the Oculus Rift is finally complete - The Verge, The Verge. Available at: https://www.theverge.com/2016/12/5/13811232/oculustouch-rift-vr-motion-controller-review (Accessed: 29 May 2018).

34. Shapiro, R. B. and Ahrens, M. (2016) 'Beyond blocks: syntax and semantics', Communications of the ACM, 59(5), pp. 39-41.

35. Slade, D. (2018) Black Mirror: Bandersnatch. Netflix.

36. Solon, O. (2018) Google's robot assistant now makes eerily lifelike phone calls for you | Technology | The Guardian. Available at: https://www.theguardian.com/technology/2018/may/08/google-duplex-assistant-phonecalls-robot-human (Accessed: 29 May 2018).

37. Turing, A. (1950) 'Computing Machinery and Intelligence', Mind, 59(236), pp. 433-460. doi: https://doi.org/10.1093/mind/LIX.236.433.

38. Twirlbound (2019) 'Pine'. Breda, Netherlands: Twirlbound.

39. Valls-Vargas, J., Zhu, J. and Ontañón, S. (2017) 'From computational narrative analysis to generation', Proceedings of the International Conference on the Foundations of Digital Games - FDG '17, pp. 1-4. doi: 10.1145/3102071.3106362.

40. Verbrugge, C. (2003) 'A Structure for Modern Computer Narratives', International Conference on Computers and Games. Springer, pp. 308-325. doi: 10.1007/978-3-54040031-8 21.

41. Vonnegut, K. (2004) 'Shape of Stories'. Available at: https://www.youtube.com/watch?v=GOGru_4z1Vc.

42. Weber, T. (2016) Late Shift. Switzerland.

43. Yannakakis, G. N. and Togelius, J. (2015) 'A Panorama of Artificial and Computational Intelligence in Games', IEEE Transactions on Computational Intelligence and AI in Games, 7(4), pp. 317-335. doi: 10.1109/TCIAIG.2014.2339221.

44. Yannakakis, G. N. and Togelius, J. (2018) Artificial Intelligence and Games. Springer.

45. Zhu, J. and Harrell, D. F. (2009) 'Memory, Reverie Machine: towards a dance of agency in interactive storytelling', Proceedings of the International Symposium on Electronic Art (ISEA) Conference. 